\title{
FATORES DETERMINANTES DOS CUSTOS DOS TRATAMENTOS PARA IDOSOS COM FRATURA DE QUADRIL
}

\section{Determining factors in the costs of treatments for elderly people with hip fracture}

\author{
Fatima Izabel Dornelles Farias ${ }^{\mathrm{a}}$, Newton Luiz Terraa, Renata Lenz de Brumb, \\ Franciele Alves ${ }^{b}$, Carolina de Souza Frare ${ }^{b}$, Marcelo Teodoro Ezequiel Guerra ${ }^{b}$
}

OBJETIVO: Avaliar os fatores associados aos custos hospitalares no tratamento de idosos com fratura de quadril (FQ) em um hospital público. MÉTODOS: Foi realizado um estudo de coorte retrospectivo de prontuários de um hospital público da Região Metropolitana de Porto Alegre (RS). Foram incluídos 134 prontuários de idosos com diagnóstico de FQ, internados no período de julho de 2014 a outubro de 2016. RESULTADOS: A idade dos idosos variou de 61 a 96 anos, com média de 79,4 anos ( \pm 8,8). Houve predominância do gênero feminino (73,1\%). O tempo médio de internação hospitalar foi de 13,87 dias. Na avaliação da presença de morbidades associadas, 56 (41,7\%) apresentaram pelo menos uma morbidade, e 64 (47,7\%), de duas a seis morbidades associadas. 0 custo médio das internações por paciente foi de $R \$ 9.390,21$ (US\$2.745). Na composição geral dos custos, os serviços de enfermagem, nutrição, hotelaria e limpeza, materiais, medicamentos e diárias representaram 78,3\% dos gastos, seguidos pelas órteses e próteses (10,7\%) e pelos honorários médicos (10\%). Ocorreu associação significativa entre o tempo para iniciar a cirurgia e a média de permanência ( $p<0,001$ ), entre o tempo para dar início à cirurgia e os custos hospitalares $(p<0,001)$, e entre as comorbidades e os custos hospitalares ( $p<0,001)$. CONCLUSÃO: Além dos custos diretos da assistência, foram identificados como fatores associados aos custos o número de morbidades, a média de permanência e o tempo para iniciar a cirurgia. Quanto maior o tempo entre a fratura e a cirurgia, maior foi o custo total.

PALAVRAS-CHAVE: idoso; custos; fraturas do quadril.

OBJECTIVE: To evaluate associated factors with hospital costs in the treatment of elderly patients with hip fracture in a public hospital. METHODS: A retrospective cohort study of medical records from a public hospital in the metropolitan area of Porto Alegre (RS) was performed. They included 134 medical records of elderly patients with hip fracture diagnosis, hospitalized from July 2014 to October 2015. RESULTS: The age of patients ranged from 61 to 96 years old, with an average of 79.4 years ( \pm 8.8 ). There was a predominance of the female gender (73.1\%). The average length of hospital stay was 13.87 days. In assessing the presence of associated morbidities, 56 (41.7\%) had at least one morbidity, and 64 (47.7\%) had two to six associated morbidities. The average cost of hospitalization per patient was R $\$ 9.390 .21$ (US $\$ 2,745$ ). At the general composition of costs, nursing and nutrition services, hostelry and cleaning materials, medicines and daily expenses accounted for $78.3 \%$ of the expenditures, followed by orthotics and prosthetics (10.7\%) and medical fees (10\%). There was a significant association between the time to start the surgery and the average length of stay $(p<0.001)$, between the time to start the surgery and hospital costs $(p<0.001)$, and between the sum of morbidity and hospital costs $(p<0.001)$. CONCLUSION: In addition to the direct costs, the comorbidities, the average residence time and the time to start the surgery have been identified as factors associated with the costs. The longer the time to start the surgery, the higher was the total cost.

KEYWORDS: aged; costs; hip fractures. 


\section{INTRODUÇÃO}

Nos idosos são comuns e frequentes doenças mais graves, levando-os ao declínio funcional, à morbidade e à mortalidade. ${ }^{1}$ Entre essas doenças mais graves que afetam tais pacientes está um evento agudo de grandes proporções: a fratura de quadril (FQ) de baixo impacto, decorrente de queda. O risco para esse tipo de fratura dobra a cada década após os 50 anos, e a cada ano a incidência aumenta, tornando-se um grave problema de saúde pública, com incremento dos atendimentos hospitalares em todo o mundo. ${ }^{2}$

Conforme o Ministério da Saúde do Brasil, ${ }^{3}$ 90\% dos recursos destinados à ortopedia estão absorvidos por 9 enfermidades, sendo uma delas a fratura proximal de fêmur. Estudos mostram que somente $25 \%$ dos pacientes conseguem retornar às atividades normais, $40 \%$ não são mais capazes de viver independentemente, e $20 \%$ vão a óbito após o primeiro ano da lesão, em função do agravamento de enfermidades preexistentes. ${ }^{4,5}$

A fratura proximal de fêmur está entre as lesões traumáticas mais frequentes na população idosa. Osteoporose, desnutrição, musculatura enfraquecida, diminuição das atividades da vida diária e redução da visão e dos reflexos são alguns fatores que estão relacionados com a alta incidência da fratura. ${ }^{6}$

A maioria dos pacientes beneficia-se com a estabilização cirúrgica da fratura. A fixação cirúrgica precoce é recomendada ainda nas primeiras 24 horas, sendo aceito pela literatura o prazo de até 48 horas. Estudos demonstram que o tempo para início da cirurgia a partir do momento do trauma é determinante para os resultados em termos de morbidade, mortalidade e custos. ${ }^{7}$ Quanto mais cedo os pacientes são tratados, menos tempo eles têm para desenvolver doenças iatrogênicas com resultados negativos.

Tendo em vista que essas fraturas são mais incidentes em idosos, a prevalência de morbidades e condições de fragilidade é alta entre os pacientes hospitalizados com esse diagnóstico. ${ }^{7}$ Trata-se de um perfil de paciente com alto risco para desenvolver complicações como delirium, infecções e outros eventos iatrogênicos, que por sua vez podem levar ao declínio funcional, à necessidade de cuidados em longo prazo e à morte, além de ampliar os custos totais finais dos tratamentos. ${ }^{8,9}$

Quando analisados os custos, a FQ torna-se um grande motivo de preocupação para todo o sistema de saúde. ${ }^{10}$ De modo geral, as doenças prevalentes da terceira idade tornam a assistência à saúde dessa população de três a sete vezes mais cara que o custo médio do restante praticado, sendo aquela prestada pelos hospitais a mais onerosa no montante dos gastos e a de maior risco. ${ }^{10}$ Os idosos apresentam permanência hospitalar mais elevada que as demais faixas etárias, maior exposição a fatores de risco e mais efeitos adversos decorrentes de intervenções empregadas. ${ }^{11}$
Com essa orientação, o objetivo geral deste estudo foi avaliar os custos hospitalares de tratamento de idosos com FQ em um hospital público e seus respectivos fatores determinantes.

\section{MÉTODOS}

Foi realizado um estudo de coorte retrospectivo de 134 prontuários de pacientes tratados cirurgicamente para FQ em um hospital público, no período de julho de 2014 a outubro de 2015. Considerou-se FQ as fraturas da extremidade proximal do fêmur, do colo do fêmur e as fraturas da região transtrocantérica.

Os prontuários dos pacientes internados no período de investigação com fratura proximal de fêmur foram avaliados quanto à elegibilidade para o estudo. $\mathrm{O}$ critério de exclusão adotado foi: pacientes com menos de 60 anos de idade ou com fraturas não decorrentes de queda.

O objetivo do trabalho consistiu em analisar os custos totais e médios decorrentes do tratamento cirúrgico disponibilizado aos idosos com FQ durante a internação, bem como os principais fatores determinantes desses custos.

Para a composição de tais gastos, foram eleitos grupos de apropriação contábil para os custos diretos, ou seja, todo o custo relacionado diretamente à assistência do paciente (custos da sala de emergência, procedimento cirúrgico, sala de cirurgia, serviços médicos e de enfermagem, reabilitação intra-hospitalar, materiais, medicamentos, órteses e próteses); e os custos das diárias de internação, contemplando hotelaria, higienização e alimentação. Por se tratar de um hospital público, as próteses utilizadas ali estão compatíveis com as autorizadas pelo Sistema de Gerenciamento da Tabela de Procedimentos (SIGTAP) e Órteses, Próteses e Materiais Especiais (OPME) do Departamento de Informática do Sistema Único de Saúde (DATASUS).

Não se consideraram os custos indiretos, isto é, serviços administrativos (de apoio e terceiros), nem os custos das transações. Os custos analisados foram calculados por paciente e depois agrupados em: custo médio total, custo médio por faixas de média de permanência, custo médio por faixas de tempo para iniciar a cirurgia (até dois, três e quatro dias, de cinco a dez dias e mais que dez dias), custo total de todos os tratamentos e desvio padrão (DP), todos expressos em real ( $\mathrm{R} \$$ ) e convertidos em dólar (US\$3,42) — valor médio de junho de 2016.

Para avaliar os fatores determinantes da composição total e média dos custos, além dos custos diretos, avaliaram-se as variáveis associadas ao tempo decorrente entre a fratura e o início da cirurgia (em dias), a média de permanência (em dias) e as complicações pós-operatórias. Todos os dados relativos aos custos foram retirados da ferramenta de gestão do hospital (Business Intelligence - BI), que trata os dados na base do software para gestão hospitalar MV 2000, e da plataforma P. I. Works. 
Utilizando diretrizes válidas, foi convencionado como tempo ideal entre a fratura e o início da cirurgia o período de 48 horas, que para efeito de tratamento dos dados foi convertido em dias (2 dias). As complicações pós-operatórias pesquisadas foram delirium, tromboembolismo pulmonar (TEP), trombose venosa profunda (TVP), infecção de sítio cirúrgico (ISC), pneumonia, infecção de trato urinário (ITU), úlcera por pressão (UP), insuficiência renal aguda (IRA), infarto agudo do miocárdio (IAM), acidente vascular cerebral (AVC) e hemorragia digestiva.

Com a finalidade de caracterizar o perfil dos idosos tratados, foram coletados dados sociodemográficos e epidemiológicos. Entre os dados sociodemográficos investigados estavam: idade (acima de 60 anos), gênero e hábitos de vida (tabagismo e prática de exercícios). Os dados epidemiológicos investigados foram relativos à presença de morbidades: doença arterial coronariana (DAC), AVC, diabetes mellitus (DM) tipo II, hipertensão arterial sistêmica (HAS), dislipidemia, doença pulmonar obstrutiva crônica (DPOC) e insuficiência renal crônica (IRC). A mortalidade intra-hospitalar dos pacientes tratados também foi calculada. Para mais assertividade e fidedignidade dos dados, vários instrumentos, além do prontuário informatizado (MV 2000), foram confrontados, entre eles os formulários de protocolo da enfermagem (histórico e diagnóstico de enfermagem), os formulários da avaliação nutricional e os formulários do protocolo de atendimento do serviço social e da psicologia.

Para a análise estatística, descreveram-se as variáveis categóricas relativas ao perfil de morbidades por frequências absoluta e relativa. Para comparar as variáveis numéricas entre o grupo com início de cirurgia até 48 horas (2 dias) e os demais, com tempo maior que 48 horas, o teste de Mann-Whitney foi utilizado. Na comparação de proporções, os testes do $\chi^{2}$ de Pearson ou exato de Fisher foram aplicados. Para as variáveis politômicas (mais de duas categorias), o teste dos resíduos ajustados foi usado para localizar as diferenças significativas apontadas pelo teste do $\chi^{2}$. O nível de significância adotado foi de $5 \%(p<0,05)$, e as análises foram realizadas no programa SPSS versão 21.0.

O estudo foi aprovado pelo Comitê de Ética em Pesquisa da Pontifícia Universidade Católica do Rio Grande do Sul (PUCRS), sob número CAAE 51213715.5.0000.5336.

\section{RESULTADOS}

Foram analisados 134 prontuários de pacientes idosos com fratura proximal de fêmur, sendo 98 (73,1\%) mulheres e 36 $(26,9 \%)$ homens. A idade mínima foi de 61 anos e a máxima de 97 , com média de 79,4 anos $( \pm 8,8)$. Na classificação por tipos de fratura, foram encontradas na amostra 83 (62\%) fraturas transtrocantéricas e 51 (38\%) fraturas de colo de fêmur.
Quando examinada a presença de morbidades, de acordo com os prontuários averiguados, as morbidades mais incidentes foram a HAS, o DM tipo II e o AVC prévio, com $84(62,7 \%), 24(17,9 \%)$ e $24(17,9 \%)$ pacientes, respectivamente. As demais morbidades tiveram incidências menores: 11 idosos (8,2\%) com dislipidemia; 10 (7,5\%) com DAC; 13 (9,7\%) com DPOC; 6 (4,5\%) com IRC; e 9 (6,7\%) com insuficiência cardíaca congestiva.

$\mathrm{Na}$ avaliação da presença ou não de mais de uma morbidade associada, $56(41,7 \%)$ pessoas apresentaram pelo menos uma morbidade, e 64 (47,7\%), de 2 a 6 morbidades associadas. $\mathrm{Na}$ análise da amostra houve associação positiva estatisticamente significativa entre o número de comorbidades e os dias de permanência no hospital ( $\mathrm{p}<0,001)$. Quanto maior o número de comorbidades, maior foi o período de permanência no hospital.

Quanto aos hábitos de vida, $38(28,4 \%)$ pacientes disseram praticar exercícios regularmente e 17 (12,7\%) deles declararam ser tabagistas (Tabela 1). A taxa de óbito intra-hospitalar foi de $6,7 \%$, e todos os pacientes que foram a óbito tinham de duas a seis morbidades associadas.

Tabela 1 Distribuição por gênero, idade, faixa etária e morbidades.

\begin{tabular}{|c|c|}
\hline Variáveis & $\begin{array}{c}\text { Total }(\mathrm{n}=134) \\
\mathrm{n}(\%)\end{array}$ \\
\hline \multicolumn{2}{|l|}{ Sexo } \\
\hline Feminino & $98(73,1)$ \\
\hline Masculino & $36(26,9)$ \\
\hline \multicolumn{2}{|l|}{ Faixa etária (em anos) } \\
\hline 60 a 70 & $17(12,7)$ \\
\hline 71 a 80 & $48(35,8)$ \\
\hline 81 a 90 & $49(36,6)$ \\
\hline 91 ou mais & $20(14,9)$ \\
\hline \multicolumn{2}{|l|}{ Morbidades } \\
\hline DM & $24(17,9)$ \\
\hline HAS & $84(62,7)$ \\
\hline Dislipidemia & $11(8,2)$ \\
\hline DAC & $10(7,5)$ \\
\hline AVC & $24(17,9)$ \\
\hline DPOC & $13(9,7)$ \\
\hline IRC & $6(4,5)$ \\
\hline Insuficiência cardíaca congestiva & $9(6,7)$ \\
\hline \multicolumn{2}{|l|}{ Hábitos de vida } \\
\hline Prática de exercícios & $38(28,4)$ \\
\hline Tabagismo & $17(12,7)$ \\
\hline
\end{tabular}

DM: diabetes mellitus; HAS: hipertensão arterial sistêmica; DAC: doença arterial coronariana; AVC: acidente vascular cerebral; DPOC: doença pulmonar obstrutiva crônica; IRC: insuficiência renal crônica. 
A média geral dos custos da internação foi de $\mathrm{R} \$ 9.390,21$ (US\$2.745,67). Quando estratificado por tipo de internação, para pacientes que utilizaram a estrutura da área cirúrgica e as enfermarias, o custo foi de $\mathrm{R} \$ 6.411 .30$ (US\$1.874), entretanto os pacientes que recorreram, além da estrutura cirúrgica e das enfermarias, também à UTI custaram, em média, R\$15.556,85 (US\$ 4.548). O custo médio das órteses e próteses foi de R $\$ 1.015,77$ (US\$296). O menor custo de internação foi de R $\$ 3.327,34$ (US\$ 972), e o maior, de R $\$ 61.669,58$ (US\$18.032).

O hospital pesquisado teve custo aproximado para o tratamento dos 134 pacientes participantes deste estudo, acometidos por fratura proximal de fêmur, de $\mathrm{R} \$ 929.630,84$ (US\$271.821), sem indexador de correção. Considerando a composição geral dos custos, 78,3\% deles foram apropriados a diárias (serviços de enfermagem, nutrição, hotelaria e limpeza) e materiais e medicamentos; $10,7 \%$, a órteses e próteses; e $10 \%$, a honorários médicos.

$\mathrm{Na}$ análise do tempo decorrido entre a fratura e o início da cirurgia, o menor tempo foi de 24 horas (1 dia), e o maior, 19 dias. O tempo médio para começar a cirurgia foi de 6,98 dias $( \pm 5)$. O tempo de internação hospitalar variou de 4 a 92 dias, com média de permanência no hospital de 13,87 dias, e 61 (45,5\%) das internações ultrapassaram 10 dias.

$\mathrm{Na}$ avaliação dos fatores determinantes dos custos, além da apropriação dos custos diretos e das diárias, houve associação estatisticamente significativa entre o tempo para o início da cirurgia e o custo total ( $\mathrm{p}<0,001)$. Quanto maior o tempo para dar início à cirurgia, maior foi o custo total. Também houve associação entre o tempo para início da cirurgia e a média de permanência no hospital $(p<0,001)$ (Tabela 2).
Quanto maior o tempo para iniciar a cirurgia, maior foi o número de dias de internação.

As maiores contas hospitalares foram atribuídas a pacientes com intervalo maior de tempo entre a fratura e o início da cirurgia - mais de sete dias de internação —, mais de duas morbidades e complicações no pós-operatório. Pacientes que realizaram tardiamente a cirurgia foram mais onerosos que aqueles que fizeram a cirurgia em até dois dias $(\mathrm{p}<0,001)$ após a fratura. Esse grupo de pacientes também foi o que apresentou o maior número de complicações no pós-operatório, sendo as complicações mais incidentes o delirium, a IRA e a ITU, seguidas de pneumonia e de ISC. Entretanto, não se constatou associação estatística entre o número de complicações e a média de permanência no hospital. A idade não mostrou relação com os maiores custos ( $p$ > 0,05); a idade média do grupo de pacientes com os maiores custos foi a mesma de todo o grupo pesquisado.

\section{DISCUSSÃO}

Os resultados do estudo demonstram que o tratamento de idosos com fratura proximal de fêmur tem grande impacto para os custos totais do hospital, tanto pelo valor médio dos tratamentos disponibilizados como pela representatividade na escala de produção. Entretanto, percebe-se que fatores associados, tais como o tempo para iniciar a cirurgia, a média de permanência no hospital e o perfil de comorbidades, são determinantes para os custos finais dos tratamentos. Para melhor compreensão dessas relações, é preciso analisar separadamente todos os fatores envolvidos.

Tabela 2 Análise dos fatores determinantes dos custos totais .

\begin{tabular}{|c|c|c|c|}
\hline \multirow{2}{*}{ Variáveis } & \multirow{2}{*}{$\mathbf{n}$} & Custo total (R\$) & \multirow{2}{*}{$\mathbf{p}^{* *}$} \\
\hline & & Mediana (P25 - P75) & \\
\hline \multicolumn{3}{|c|}{ Tempo para iniciar a cirurgia (em dias) } & \multirow{6}{*}{$<0,001$} \\
\hline Até 2 & 30 & $5.118(3.985-6.010)^{*}$ & \\
\hline 3 & 12 & $6.397(5.242-7.609)^{*}$ & \\
\hline 4 & 13 & $6.005(5.242-8.533)^{*}$ & \\
\hline 5 a 10 & 46 & $8.592(7.369-9.250)^{*}$ & \\
\hline 11 ou mais & 33 & $13.971(11.267-21.385)^{*}$ & \\
\hline \multicolumn{3}{|c|}{ Dias de permanência } & \multirow{6}{*}{$<0,001$} \\
\hline$\leq 5$ & 09 & $4.154(3.813-5.015)^{*}$ & \\
\hline 6 a 10 & 64 & $6.392(5.728-7.076)^{*}$ & \\
\hline 11 a 15 & 31 & $8.790(8.351-9.099)^{*}$ & \\
\hline$>15$ & 30 & $13.497(11.212-20.936)^{*}$ & \\
\hline Total & 134 & & \\
\hline
\end{tabular}

*Não diferem pelo teste de Dunn a 5\% de significância; ${ }^{* *}$ teste de Kruskal-Wallis. 
O perfil dos idosos com fratura proximal de fêmur encontrado nos registros avaliados neste estudo está bastante próximo dos resultados disponibilizados no banco de dados da American Academy of Orthopaedic Surgeons (AAOS), ${ }^{2}$ da British Orthopaedic Association (BOA) ${ }^{12}$ e do Ministério da Saúde do Brasil. ${ }^{3}$ Esses dados mostram que existe a prevalência do gênero feminino nos grupos de pacientes com FQ decorrente de queda. Na presente investigação não foi diferente. Houve predominância do gênero feminino $(73,1 \%)$ em relação ao masculino (26,9\%). ${ }^{6}$ A maior incidência de FQ em mulheres pode estar relacionada ao fato de o sexo feminino ter maior expectativa de vida e, portanto, estar mais exposto a fatores de risco, iniciar o processo de declínio de massa óssea antes do homem, ter maior suscetibilidade para quedas, além das influências antropométricas e dos fatores genéticos associados ao sexo. ${ }^{7,13}$

Quando avaliada a média de idade, o resultado desta pesquisa foi de 79,4 anos $( \pm 8,8)$, maior em comparação aos estudos feitos por Hungria Neto, Dias e Almeida, ${ }^{14}$ com média de 75,8 anos, mas menor que o valor encontrado no banco de dados dos estudos epidemiológicos do Ministério da Saúde, ${ }^{3}$ que fizeram referência à média de 83 anos. Todavia, os resultados aferidos estão em consenso com esta e outras análises, quando identificaram que há incidência maior de idosos com FQ na sétima e na oitava década de vida. ${ }^{5,7} \mathrm{~A}$ fratura proximal de fêmur acomete idosos mais longevos, porque as alterações musculoesqueléticas podem comprometer o desempenho de habilidades motoras, dificultar a adaptação dos indivíduos ao ambiente e predispô-los às quedas de própria altura. ${ }^{15}$

$\mathrm{Na}$ classificação por tipos de fratura foram encontradas na amostra 83 (62\%) fraturas transtrocantéricas e 51 (38\%) fraturas de colo de fêmur, quantidades condizentes com a literatura. ${ }^{1,6,8,14}$

A prevalência de comorbidades é alta entre os pacientes hospitalizados com FQ. ${ }^{7}$ Idosos com fraturas por queda têm alta prevalência de comorbidades e elevado risco para complicações. ${ }^{16}$ Os dados encontrados neste estudo apontam para a prevalência de HAS, DM tipo II e AVC prévio, com 84 (62,6\%), 24 (17,9\%) e 24 (17,9\%) pacientes, respectivamente. Quanto maior o número de comorbidades, maior foi o tempo de internação, e houve associação estatisticamente significativa entre o número de comorbidades e os dias de permanência no hospital ( $\mathrm{p}<0,001)$. Essa associação remete a custos hospitalares mais elevados, tanto pela necessidade de manejo e estabilização de quadros clínicos concomitantemente ao tratamento da fratura como pelo alto risco para complicações. ${ }^{16}$ Os resultados apresentados corroboram informações disponibilizadas no banco de dados de outros países ${ }^{5,16}$ e em estudos do Brasil. ${ }^{6,8}$ Mesquita et al. ${ }^{17}$ fazem referência à maior prevalência de doenças cardíacas.
A taxa de mortalidade durante a internação foi de 6,7\%. Também nos estudos de Ginsberg, Adunsky e Rasooly ${ }^{10} \mathrm{e}$ Thomas et al. ${ }^{18}$ as taxas de mortalidade praticadas foram entre 6,5 e 8,6\%, respectivamente. Esses mesmos estudos exibiram associação significativa entre menos tempo para a intervenção cirúrgica, menos tempo de permanência, menor taxa de mortalidade e menores custos hospitalares.

A análise dos prontuários dos pacientes internados no hospital demonstrou que $\mathrm{R} \$$ 929.630,84 (US\$271.821) foram disponibilizados para tratamento dos pacientes durante o período de tratamento hospitalar.

$\mathrm{Na}$ presente investigação, apropriados todos os custos diretos e indiretos propostos no escopo de pesquisa, a média de custos da internação foi de R \$9.390,21 (US\$2.745,67). Quando utilizados os serviços da unidade de terapia intensiva (UTI), a média de custos subiu para R $\$ 15.556,85$ (US\$ 4.548,78).

Bracco et al. ${ }^{19}$ utilizaram as mesmas medidas de apropriação dos custos em um estudo acerca de um hospital universitário de São Paulo, no Brasil. Esse estudo contabilizou média de custos para a internação no valor de $\mathrm{R} \$ 8.266,25$, que convertido ao mesmo valor médio do dólar usado aqui representa US\$2.417, quantia muito semelhante aos resultados encontrados nesta pesquisa. Entretanto, quando analisada a média de custos com a UTI, os valores foram de $\mathrm{R} \$ 6.575,14$ (US\$1.922), bastante inferiores aos custos aferidos nesta investigação. Não foram descritos dados a respeito do perfil de comorbidades nem outros fatores determinantes dos custos que pudessem permitir outras comparações.

No Brasil, são comuns os estudos com a avaliação de custos associados a idosos com FQ tanto no setor público como no privado, porém na sua grande maioria os resultados estão focados nos custos para o sistema de remuneração, utilizando como base os dados fontes pagadoras (Sistema de Informações Hospitalares - SIH — do SUS e Agência Nacional de Saúde Complementar - ANS), ${ }^{20,21}$ que não retratam os custos diretos praticados com a assistência, mas sim os repasses realizados para as instituições de saúde. ${ }^{17,19} \mathrm{Na}$ comparação com estudos de outros países, há de se considerar as diferenças de abordagem e organização dos tratamentos. Esses estudos frequentemente analisam os custos acompanhando os tratamentos até a reabilitação (um ou dois anos de seguimento), embora sejam levados em conta os custos com o tratamento inicial (hospitalar), o mais oneroso e definidor deles, com a reabilitação e/ou as readmissões. ${ }^{19}$ Quando se averigua a média de custos com internações, ${ }^{18}$ as faixas médias praticadas são bem maiores do que no Brasil. Provavelmente tal incremento corresponda a valores atribuídos a custos com unidades de reabilitação geriátrica, que não é a realidade do Brasil, onde o paciente faz a reabilitação no âmbito ambulatorial. Burgers et al., ${ }^{22}$ na Holanda, atribuíram média de 
custos de $€ 5$ 5.732. Outra investigação americana apontou para o custo médio da internação de US $\$ 559$ (por dia), com média de permanência no hospital de 14 dias (US\$ 7.826). ${ }^{10}$

Entretanto, se analisados os fatores determinantes dos custos, um grande número de estudos e de experiências exitosas em outros países aponta a cirurgia precoce como um fator importante para a melhoria da qualidade dos tratamentos com custo-efetividade. ${ }^{7,10}$ No presente estudo, os resultados demonstraram igualmente que o tempo para iniciar a cirurgia é um fator determinante, significativo estatisticamente, para o aumento dos custos dos tratamentos e da média de permanência hospitalar. Os pacientes que realizaram a cirurgia mais precocemente tiveram menores custos e menos tempo de internação.

Em centros de tratamento especializados dos Estados Unidos $^{2,5}$ da Inglaterra ${ }^{7}$ e do Reino Unido ${ }^{7}$ programas são desenvolvidos para que diretrizes possam ser aplicadas na busca de melhores desfechos para os tratamentos. Duas diretrizes foram incorporadas e chamam a atenção: o "comanejo" geriátrico, ${ }^{7,16} \mathrm{com}$ profissionais clínicos e/ou geriatras, cirurgiões e enfermeiros especialistas atuando conjuntamente para a abordagem desses pacientes, como forma de preservar as condições de saúde do idoso; e a antecipação da cirurgia, como forma de reduzir os riscos e estimular a rápida mobilização. ${ }^{16}$ A expertise alcançada em tais modelos registra resultados qualificados na redução da mortalidade, da permanência hospitalar, das complicações pós-operatórias e dos custos hospitalares, com impactos inclusive nos custos do seguimento até dois anos. Nos estudos de Ginsberg, Adunsky e Rasooly ${ }^{10}$ e Thomas et al., ${ }^{18}$ esses resultados também indicaram associação significativa entre o menor tempo para a intervenção cirúrgica e a média de permanência hospitalar, com menores taxas de mortalidade e menores custos hospitalares.

Por seu lado, esta investigação apresenta algumas limitações, que servem de estímulo para o aprofundamento de pesquisas futuras. Trata-se de um estudo retrospectivo, examinado com base em registros assistenciais e administrativos. A dependência da qualidade desses registros pode trazer vieses que por ora não são passíveis de detecção. Este estudo considerou uma amostra de 134 pacientes somente no período da internação. Estudos de seguimento podem melhor traduzir os custos totais implicados nesse tipo de trauma no idoso.
No tocante aos fatores associados ao custo, não foram abordados fatores relacionados à eficiência operacional. $\mathrm{O}$ referido tipo de análise somente seria possível em estudos prospectivos.

\section{CONCLUSÃO}

A FQ em idosos é uma doença grave e frequente, e sua incidência duplica a cada década. O conhecimento acerca dos custos envolvidos nos tratamentos desses indivíduos e dos fatores associados a tais custos é muito relevante, não somente para os estudos do envelhecimento, mas para o poder público e as instituições, uma vez que ambos oferecem subsídios para o planejamento das ações em saúde.

O presente estudo demonstrou que o monitoramento dos custos não deve se restringir somente à análise dos custos envolvidos diretamente nos cuidados prestados ao paciente, mas abranger vários outros fatores que podem ser determinantes para os custos globais. Os achados aqui mostraram que o tempo entre a fratura e a cirurgia está vinculado a maiores custos hospitalares e maior tempo de internação. Idosos com tempo de internação prolongada estão mais expostos a iatrogenias, que por sua vez implicam maiores custos para toda a linha de cuidado. Mediante a constatação de que as comorbidades indicaram associação com maiores custos, o controle e a estabilização das doenças prévias relacionadas à fratura são fundamentais para a cadeia geral dos custos.

De acordo com os achados e corroborando a literatura, existe associação significativa, em uma relação de causa e efeito, entre a tomada de decisão/prontidão para o tratamento cirúrgico precoce, a média de permanência hospitalar e a soma de morbidades, o que impacta no resultado final da assistência e dos custos. De posse dessa informação, há de se pensar em novas e mais eficientes formas de abordagens cirúrgica e clínica direcionadas para esses fatores. Modelos assistenciais colaborativos e protocolos de acesso e de tratamento parecem ser o caminho para o maior custo-efetividade desses tratamentos.

\section{CONFLITO DE INTERESSES}

Os autores declaram não haver conflito de interesses.

\section{REFERÊNCIAS}

1. Eriksson M, Kelly-Pettersson P, Stark A, Ekman AK, Skoldenberg O. "Straight to bed" for hip-fracture patients: a prospective observational cohort study of two fast-track systems in 415 hips. Injury. 2012;43(12):2126-31. DOI: 10.1016/j.injury.2012.05.017.
2. American Academy Of Orthopaedic Surgeons. Management Of hip fractures in the elderly. Evidence- based clinical practice guideline. Rosemont: American Academy of Orthopaedic Surgeons; 2014 
3. Brasil. Ministério da Saúde. Departamento de Ciência e Tecnologia, Secretaria de Ciência, Tecnologia e Insumos Estratégico. ELSA Brasil: maior estudo epidemiológico da América Latina. Rev Saúde Pública. 2009;43(1):1-2.

4. Albuquerque ALM, Souza Filho PGT, Braga Junior MB, Cavalcante Neto JS, Medeiros BBL, Lopes MBG. Epidemiologia das fraturas em pacientes do interior do Ceará tratadas pelo SUS. Acta Ortop Bras. 2012;20(2):66-9. DOI: http://dx.doi.org/10.1590/S1413-78522012000200001.

5. Royal College of Physicians. Falls and Fragility Fracture Audit Programme (FFFAP). National Hip Fracture Database (NHFD) extended report 2014. London, England: Royal College of Physicians; 2014.

6. Muniz CF, Arnaut AC, Yoshida M, Trelha CS. Caracterização dos idosos com fratura de fêmur proximal atendidos em hospital escola público. Espaço Saúde. 2007:8(2):33-8.

7. Friedman SM, Mendelson DA, Bingham KW, Kates SL. Impact of a comanaged geriatric fracture center on short-term hip fracture outcomes. Arch Intern Med. 2009;169(18):1712-7. DOI: 10.1001/ archinternmed.2009.321.

8. Bittar CK, Dota S, Zabeu JL. Perfil epidemiológico das fraturas do fêmur proximal no paciente idoso. Rev Ciênc Méd. 2004;13(3):235-9.

9. Machado FN, Machado AN, Soares SM. Comparação entre a capacidade e desempenho: um estudo sobre a funcionalidade de idosos dependentes. Rev Latino-Am Enfermagem. 2013;21(6):1321-9. DOI: 10.1590/01041169.2682.2370.

10. Ginsberg G, Adunsky A, Rasooly I. A cost-utility analysis of a comprehensive orthogeriatric care for hip fracture patients, compared with standard of care treatment. Hip Int. 2013;23(6):570-5. DOI: 10.5301/hipint.5000080.

11. Loyola Filho AI, Matos DL, Giatti L, Afradique ME, Peixoto SV, Lima-Costa MF. Causas de internações hospitalares entre idosos brasileiros no âmbito do Sistema Único de Saúde. Epidemiol Serv Saúde. 2004;13(4):229-38. DOI: http://dx.doi.org/10.5123/S167949742004000400005.

12. British Orthopaedic Association (BOA). More patients surviving due to continued improvements in hip fracture care. Medical News Today. MediLexicon, Intl., 12 Sep. 2014 [cited 2016 Aug 26]. Available from: http://www.medicalnewstoday.com/releases/282356.php
13. Barros IFO, Pereira MB, Weiller TH, Anversa ETR. Internações hospitalares por quedas em idosos brasileiros e os custos correspondentes no âmbito do Sistema Único de Saúde. Kairós Gerontologia. 2015;18(4):63-80.

14. Hungria Neto JS, Dias CR, Almeida JDB. Características epidemiológicas e causas da fratura do terco proximal do fêmur em idosos. Rev Bras Ortop. 2011;46(6):660-7. DOI: http://dx.doi.org/10.1590/S010236162011000600007

15. Arndt ABM, Telles JL, Kowalski SC. O custo direto da fratura de fêmur por quedas em pessoas idosas: análise no setor privado de saúde na cidade de Brasília. Rev Bras Geriatr Gerontol. 2011;14(2):221-31.

16. Mendelson DA, Friedman SM. Principles of comanagement and the geriatric fracture center. Clin Ger Med. 2014;30(2):183-9. DOI: 10.1016/j.cger.2014.01.016.

17. Mesquita GV, Lima MALTA, Santos AMR, Alves ELMA, Brito JNPO, Martins MCC. Morbimortalidade em idosos por fratura proximal do fêmur. Texto Contexto Enferm. 2009;18(1):67-73. DOI: http://dx.doi org/10.1590/50104-07072009000100008.

18. Thomas CJ, Smith RP, Uzoigwe CE, Braybrooke JR. The weekend effect: short-term mortality following admission with a hip Fracture. Bone Joint J. 2014;96-B(3):373-8. doi: 10.1302/0301-620X.96B3.33118.

19. Bracco OL, Fortes EM, Raffaelli MP, Araújo DV, Santili C, Lazaretti-Castro M. Custo hospitalar para tratamento da fratura aguda do fêmur por osteoporose em dois hospitais-escola conveniados ao Sistema Único de Saúde. J Bras Econ Saúde. 2009(1);1:3-10.

20. Brasil. Ministério da Saúde. Morbidade hospitalar do SUS por local de internação. Notas técnicas [Internet]. 2016 [cited 2016 May 13]. Available from: http://tabnet.datasus.gov.br/cgi/sih/midescr.htm

21. Brasil. Agência Nacional de Saúde Suplementar. Padrão para Troca de Informação de Saúde Suplementar - TISS [Internet]. 2016 [cited 2016 May 13]. Available from: http://www.ans.gov.br/prestadores/tiss-trocade-informacao-de-saude-suplementar\#sthash.K82m8AlO.dpuf

22. Burgers PTPW, Hoogendoorn M, Van Woensel EA, Poolman RW, Bhandari $\mathrm{M}$, Patka P, et al. Total medical costs of treating femoral neck fracture patients with hemi- or total hip arthroplasty: a cost analysis of a multicenter prospective study. Osteoporose Int. 2016;27(6):19992008. DOI: 10.1007/s00198-016-3484-z. 